\title{
Pragmatic Convergence and the Epistemology of an Adolescent Neuroethics
}

\author{
JOSEPH J. FINS and JUDY ILLES
}

Last year neuroethics celebrated its 15th birthday. Although the details of the field's conception are the subject of some debate, ${ }^{1}$ the birth is most frequently ascribed to a landmark meeting called "Neuroethics: Mapping the Field" that was held in San Francisco in 2002. Attended by luminaries such as William Safire and Albert Jonsen, this conference became central to a collective birth myth about origins. Indeed the birth certificate that attests to the arrival of neuroethics is the rich anthology of essays published by the Dana Foundation. ${ }^{2}$ This volume has become a touchstone for our young field.

To celebrate the important milestone marking the 15th anniversary, the leadership of the International Neuroethics Society (INS) convened a panel to assess how the field has evolved as it enters adolescence. The result is the group of derivative articles collected here, entitled "Competing Identities of Neuroethics." Expertly edited by Jennifer A. Chandler, ${ }^{3}$ who also authors a typology of emerging scholarship in neurolaw and neuroethics, the collection charts the development of the field over the past decade and a half and considers its future prospects. Chandler is joined by Tom Buller ${ }^{4}$ and the scholarly teams of Eric Racine and Matthew Sample ${ }^{5}$ and Gabriela Pavarini and Illina Singh. ${ }^{6}$

As could be anticipated, all the essayists take as their starting point Adina Roskies' distinction between the neuroscience of ethics and the ethics of neuroscience. $^{7}$ This well-worn dichotomy points to the underlying neuroscience of how ethical judgments are made as well as the ethical implications of advances in neuroscience. From the start, her formulation created a complicated bifurcation across the two-culture divide articulated by C.P. Snow. ${ }^{8}$

Neuroethics sought to reconcile, or at least better apprehend these two ways of knowing. The first way draws on the emerging knowledge of brain science; the second draws on more traditional sources in the humanities. On the one hand, there is an appeal to science and empiricism as a way to understand how we reason about ethical choices and moral quandaries. On the other hand, there is an appeal to the consequences of new neuroscience in the world, not just emerging knowledge about how we make morally laden decisions, but also about how advances in neurotechnology affect our health and sense of self. There is the prospect of more science, but at the same time a risk of reductionism and the narrowing of the gaze so prized by humanists. It is a complicated mix of inheritances.

So how has this birthright played out as neuroethics has grown and evolved? Does our field still retain the pluripotentiality that Roskies framed with a conjunction

Dr. Fins acknowledges the support of the Jerold B. Katz Foundation to Weill Cornell Medical College and the Consortium for the Advanced Study of Brain Injury (CASBI). Dr. Judy Illes is Canada Research Chair in Neuroethics. 
linking both the neuroscience of ethics and the ethics of neuroscience? Or, have these competing identities been reconciled into a coherent whole? The first would suggest two distinct strains of neuroethics. The second offers a more mature field that has integrated both aspects of neuroethics into a more coherent whole.

The answer is not yet in, but the adolescence of a discipline is a good moment to check in. Even before the advent of neuroethics, adolescence has been appreciated as a time of individuation, when the teen explores versions of future selves, often experimenting with competing identities en route to adulthood. This exploration can be a promissory note for a bright future but can also be fraught with the traumas of separation from parental influences. For most, adolescence is something to be overcome, a transition point that may be more fondly recalled than experienced. But it is a time of tremendous exploration and growth.

The same is true for neuroethics as it seeks its identity, or identities, as a discipline. But it is a complicated process of maturation as it separates from paternal disciplines such as bioethics, neuroscience, neurology, psychiatry, and the law. Are these ties that bind or constrain? Do they foster a rich inheritance or impose an enduring family resemblance? Or, has something new been created through a meiotic mix of disciplinary recombination? In a lovely phrase, Pavarini and Singh describe neuroethics as a "marriage of apparently mismatched partners."

Buller cuts to the heart of this complicated marriage covenant when he deconstructs the place of neuroscience in moral reasoning. Invoking the work of Joshua Greene, Buller notes the paradox that deontological arguments found in classical ethics are more emotional and less rational or deliberative than those found in consequentialism. This raises questions about the fate of moral intuitions as well as much of analytic philosophy. It is a sobering finding that points to the possibility of bias when we make principle-based arguments.

The Kantians will find themselves weeping. And that begs the question of what to do with emotion? Buller aptly explores these challenges with variants on the Trolley Problem. But as he pulls the reader along, he also cautions not to appeal to empiricism to answer questions that science cannot answer alone. Pavarini and Singh, invoking David Hume, remind the reader that moral judgments of right and wrong cannot be made from reason alone. Whereas these admonitions are prudent, each brings us back to the original tension embedded in Roskies' original formulation.

Fortunately, both Racine and Sample as well as Pavarini and Singh offer a remedy to this quandary. Each team of scholars fruitfully appeals to the pragmatism of John Dewey. Instead of choosing one way of knowing, they would have us avoid dichotomies and enjoin multiple ways of knowing. Racine and Sample respond to what they rightly call the "two problematic foundations of neuroethics," by calling for a Deweyan reconstruction.

Epistemic pluralism allows for the incorporation of emerging scientific knowledge, whereas findings are also contextualized into a broader societal and humanistic pane. In this way, empirical data do not lead to reductionism, or the limiting frame of scientism, ${ }^{9}$ but rather to a broader canvas by identifying what Dewey described as the recognition of the problematic situation.

In Dewey's philosophy, the recognition of the problematic situation is central to his theory of inquiry. ${ }^{10}$ In a pragmatic frame, new scientific information does not answer the question but prompts further inquiry. Instead, empirical data make it possible to recognize new normative challenges and responsibilities. For example, 
once science demonstrates that some patients with disorders of consciousness thought to be insensate can actually perceive pain, $11,12,13$ a pragmatic reconstruction can envision the neuropalliative care needs of this population. ${ }^{14}$ More broadly, the recognition of covert consciousness can also prompt discussion about the civil and disability rights of a marginalized population heretofore unidentified until science stepped in. ${ }^{15,16,17}$ As Racine and Sample note, instead of privileging science, pragmatism prompts interdisciplinarity. Indeed, it broadens rather than constrains inquiry. ${ }^{18}$

Pragmatism also brings another virtue to neuroethics. Deweyan inquiry proceeds with humility, eschewing absolutism and acknowledging that knowledge is contingent. Here, theory informs practice, and practice, in turn, informs theoretical work. This feedback loop makes pragmatism compatible with hypothesis-driven neuroscience and the assessment of emerging neurotechnologies such as neuroimaging when methods need critique ${ }^{19}$ or incidental findings addressed. ${ }^{20,21}$ A pragmatic approach allows for the assessment of these novel circumstances in a manner not achieved through the analogic reasoning offered by casuistry.

Pavarini and Singh illustrate the utility of pragmatism through the content and structure of their article. They begin with a theoretical rationale for pragmatism and conclude with a description of their empirical work studying how adolescents make moral judgments. Here the neuroscience of moral judgment is placed in dialogue with how "morality unfolds in everyday experience." Their work on adolescence is an especially fitting illustration, given the developmental moment of our field.

Pavarini and Singh's empirical work remind us that our teens are a work in progress. And it is the same when we consider the future of neuroethics. Chandler's taxonomy of neuroethics and the law makes the point. Even as new areas of inquiry are identified, more can be imagined. Like adolescence, within neurolaw there is the potential for disciplinary fluidity and the opportunity for additional self-discovery. For example, in neurolaw, the conversation could be broadened beyond forensics to consider justice issues. ${ }^{22,23}$ Chandler's careful deconstruction of neurolaw prompts recognition of the need for this pragmatic reconstruction beyond current categories.

As early proponents of a pragmatic approach in clinical ethics ${ }^{24,25}$ and the neurosciences, $, 26,27,28,29$ we are pleased to note the pragmatic convergence articulated in these fine articles by leading scholars. This portends well for the future of our field as it enters adulthood, ever grappling with a rich and complicated inheritance across the disciplines.

\section{Notes}

1. Moreno JD. The anniversary of the first neuroethics conference (no, not that one). The Neuroethics Blog. February 6, 2018; available at http:/ / www.theneuroethicsblog.com/2018/02/the-anniversaryof-first-neuroethics.html\#more (last accessed 12 Feb 2018).

2. Marcus SJ. Neuroethics: Mapping the Field. New York: The Dana Press; 2002.

3. Chandler JA. Neurolaw and neuroethics. Cambridge Quarterly of Healthcare Ethics 2018;27(4): 590-598.

4. Buller T. The new ethics of neuroethics. Cambridge Quarterly of Healthcare Ethics 2018;27(4): 558-565.

5. Racine E, Sample M. Two problematic foundations of neuroethics and pragmatist reconstruction. Cambridge Quarterly of Healthcare Ethics 2018;27(4):566-577. 


\section{Pragmatic Convergence and the Epistemology of an Adolescent Neuroethics}

6. Pavarini G, Singh I. Pragmatic neuroethics: Lived experiences as a source of moral knowledge. Cambridge Quarterly of Healthcare Ethics 2018;27(4):578-589.

7. Roskies A. Neuroethics for the new millenium. Neuron 2002;35:21-3.

8. Snow CP. The Two Cultures and The Scientific Revolution. New York: Cambridge University Press; 1959.

9. Stein DJ, Illes J. Beyond scientism and skepticism: An integrative approach to global mental health. Frontiers in Psychiatry 2015;6:166.

10. Miller FG, Fins JJ, Bacchetta MD. Clinical Pragmatism: John Dewey and clinical ethics. The Journal of Contemporary Health Law and Policy 1996;13(27):27-51.

11. Fins JJ. Neuroethics and disorders of consciousness: A pragmatic approach to neuro-palliative care. In: Laureys S, Tononi G, eds. The Neurology of Consciousness, Cognitive Neuroscience and Neuropathology. Amsterdam: Academic Press-Elsevier; 2008:234-44.

12. Chatelle C, Thibaut A, Whyte J, De Val MD, Laureys S, Schnakers C. Pain issues in disorders of consciousness. Brain Injury 2014;28(9):1202-8.

13. Naro A, Bramanti P, Bramanti A, Calabrò RS. Assessing pain in patients with chronic disorders of consciousness: Are we heading in the right direction? Conscious Cognition 2017;55:148-55.

14. Fins JJ, Pohl BR. Neuro-palliative care and disorders of consciousness. In: Hanks G, Cherny NI, Christakis NA, Fallon M, Kassa S, Portenoy RK, eds. Oxford Textbook of Palliative Medicine, $5^{\text {th }}$ Edition. Oxford: Oxford University Press; 2015:285-91.

15. Fins JJ. Rights Come to Mind: Brain Injury, Ethics and the Struggle for Consciousness. New York: Cambridge University Press; 2015.

16. Fins JJ. Brain injury and the civil right we don't think about. The New York Times. August 24, 2017; available at https:/ / www.nytimes.com/2017/08/24/opinion/minimally-conscious-brain-civilrights.html?_r=0 (last accessed 16 Feb 2018).

17. Fins JJ, Wright MS. Rights language and disorders of consciousness: A call for advocacy. Brain Injury 2018;32(5):670-4.

18. Fins JJ. Neuroethics and neurotechnology: Instrumentality and human rights. Epilogue. In: Illes J, Hossain S, eds. Neuroethics: Anticipating the Future. New York: Oxford University Press; 2017: 601-13.

19. Anderson J, Mizgalewicz Illes J. Reviews of functional MRI: The ethical dimensions of methodological critique.PloS One 2012;7(8):e42836.

20. Illes J, Borgelt E. Brain imaging: Incidental findings: In practice and in person. Nature Reviews. Neurology 2009;5(12):643-4.

21. Racine E, Northoff G, Menon RS, Kimmelman J, Illes J. A Canadian perspective on ethics review and neuroimaging: tensions and solutions. Canadian Journal of Neurological Sciences 2011;38(4): 572-9.

22. Wright MS, Varsava N, Ramirez J, Edwards K, Guevremont N, Ezer T, et al. Justice and severe brain injury: Legal remedies for a marginalized population. Florida State University Law Review, in press.

23. Eijkholt M, Anderson JA, Illes J. Picturing neuroscience research through a human rights lens: Imaging first-episode schizophrenic treatment-naive individuals. International Journal of Law and Psychiatry 2012;35(2):146-52.

24. Fins JJ, Bacchetta MD, Miller FG. Clinical Pragmatism: A method of moral problem solving. Kennedy Institute of Ethics Journal 1997;7(2):129-45.

25. Fins JJ, Miller FG, Bacchetta MD. Clinical Pragmatism: Bridging theory and practice. Kennedy Institute of Ethics Journal 1998;8(1):39-44.

26. Illes J, Raffin TA. Neuroethics: An emerging new discipline in the study of brain and cognition. Brain and Cognition 202;50(3):341-4.

27. Fins JJ. Clinical Pragmatism and the care of brain injured patients: Towards a palliative neuroethics for disorders of consciousness. Progress in Brain Research 2005;150:565-82.

28. Illes J, Chin VN. Bridging philosophical and practical implications of incidental findings in brain research. Journal of Law, Medicine, E Ethics 2008;36(2):298-304.

29. Fins JJ, Dorfman GS, Pancrazio JJ. Challenges to deep brain stimulation: A pragmatic response to ethical, fiscal, and regulatory concerns. Annals of the New York Academy of Sciences 2012;1265:80-90. 